\title{
Quartz crystal microbalance with dissipation (QCM-D) as tool to exploit antigen-antibody interactions in pancreatic ductal adenocarcinoma detection
}

\author{
Monica Bianco ${ }^{\mathrm{a}, 1}$, Alessandra Aloisi ${ }^{\mathrm{a}, 1}$, Valentina Arima ${ }^{\mathrm{a}, *}$, Michela Capello ${ }^{\mathrm{b}, \mathrm{c}}$, \\ Sammy Ferri-Borgogno ${ }^{\mathrm{b}, \mathrm{c}}$, Francesco Novelli ${ }^{\mathrm{b}, \mathrm{c}}$, Stefano Leporatti ${ }^{\mathrm{a}}$, Rosaria Rinaldi ${ }^{\mathrm{a}, \mathrm{d}}$

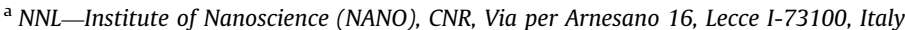 \\ ${ }^{\mathrm{b}}$ Center for Experimental Research and Medical Studies (CERMS) University of Turin San Giovanni Battista University Hospital via Cherasco 15, Turin 10126, Italy \\ ${ }^{\mathrm{c}}$ Department of Medicine and Experimental Oncology, University of Turin, Via Michelangelo 27, Torino 10125, Italy \\ d Università del Salento, Dipartimento di Matematica e Fisica "E. De Giorgi”, ex Collegio Fiorini Campus extraurbano, via per Arnesano, Lecce 73100, Italy
}

\section{A R T I C L E I N F O}

\section{Article history:}

Received 4 July 2012

Received in revised form

22 September 2012

Accepted 3 October 2012

Available online 22 October 2012

Keywords:

Quartz crystal microbalance

Self-assembled monolayer

$\alpha$-enolase

Pancreatic ductal adenocarcinoma

\begin{abstract}
A B S T R A C T
Novel synthetic peptides represent smart molecules for antigen-antibody interactions in several bioanalytics applications, from purification to serum screening. Their immobilization onto a solid phase is considered a key point for sensitivity increasing. In this view, we exploited Quartz Crystal Microbalance with simultaneous frequency and dissipation monitoring (QCM-D) with a double aim, specifically, as investigative tool for spacers monolayer assembling and its functional evaluation, as well as high sensitive method for specific immunosorbent assays. The method was applied to pancreatic ductal adenocarcinoma (PDAC) detection by studying the interactions between synthetic phosphorylated and un-phosphorylated $\alpha$-enolase peptides with sera of healthy and PDAC patients. The synthetic peptides were immobilized on the gold surface of the QCM-D sensor via a self-assembled alkanethiol monolayer. The presented experimental results can be applied to the development of surfaces less sensitive to non-specific interactions with the final target to suggest specific protocols for detecting PDAC markers with un-labeled biosensors.
\end{abstract}

(c) 2012 Elsevier B.V. All rights reserved.

\section{Introduction}

Quartz Crystal Microbalances (QCMs) are a type of acoustic wave devices that have been in use for nearly 50 years in a variety of applications, including monitoring of the thickness of metallic films deposited in vacuum. These piezoelectric tools were first thoroughly investigated in 1880 (Curie and Curie, 1880), but were not utilized as immunosensors until 1972 (Shons et al., 1972). In the past decade, QCM based immunosensors have drawn attention because of their ability to detect slight mass changes in biomaterials (Chou et al., 2002; Su and Li, 2004; Wu et al., 2003). Because of their sensitivity and compatibility with liquid-phase media, acoustic wave devices are believed to have the potential to become a useful tool in cancer screening modalities.

One of the most promising ways to reduce cancer mortality is through early detection, particularly for the most vicious of all cancer, the pancreatic cancer. Pancreatic ductal adenocarcinoma

\footnotetext{
* Corresponding author. Tel.: +00390832 298218; fax: +00390832 298230. E-mail address: valentina.arima@nano.cnr.it (V. Arima).

1 These authors contributed equally to the work.
}

(PDAC) is characterized by rapid progression, invasiveness, and resistance to treatment. The five years survival rate after the diagnosis is just 5\% (Hidalgo, 2010), mainly due to the lack of consistent markers to diagnose PDAC at an early stage. By using a serological proteome approach called SERPA we have demonstrated a new progress in the ongoing search for useful pancreatic cancer biomarkers (Tomaino et al., 2007). The glycolytic enzyme $\alpha$-enolase is up-regulated in pancreatic tumors (Cappello et al., 2009) and a careful analysis of autoantibody response to $\alpha$-enolase (ENOA) in pancreatic cancer revealed that it is associated with the disease onset (Capello et al., 2011). We have found that two acidic isoforms of ENOA (ENOA1,2) phosphorylated at Serine 419 (Zhou et al., 2010) were recognized with high frequency by most of PDAC sera (Tomaino et al., 2011). Thus, the presence of circulating autoantibodies to phosphorylated ENOA isoforms (ENOA1,2+) can efficiently discriminate, in association with the CA19.9, PDAC subjects from controls. Moreover, the presence of autoantibodies against ENOA1,2 correlated with a significantly better clinical outcome in advanced patients treated with standard chemotherapy (Novelli et al., 2011).

A crucial point in a number of immunosorbent assays is the selectivity of the specific interaction between the antibody and 
the solid-phase immobilized antigen. If the antigen is a short peptide, its immobilization to a solid support might reduce its spatial flexibility and limit or decrease the accessibility of antibody to the specific epitope. Alternative approaches able to spatially control the peptide orientation involving a spacer between the probe and the surface could overcame this constrain (Butler, 2000; Gregorius et al., 1995; Ivanov et al., 1992).

In this paper, we have developed three functionalization methods and demonstrated that one of them is more convenient than the other ones in the perspective of enhancing the sensitivity towards antigen-antibody interactions. QCM was employed to establish the less sensitive surface to un-specific interactions with the final target to suggest specific protocols for detecting PDAC markers with label-free biosensors. Furthermore, our study demonstrates that QCM with simultaneous monitoring of frequency and dissipation signals is a very sensitive tool for detecting and investigating phosphoENOAmer specific and un-specific interactions with immunoglobulins present in the sera of PDAC and healthy patients.

\section{Materials and methods}

\subsection{Chemicals}

Absolute ethanol was purchased from Carlo Erba; ammonium hydroxide (28-30\%), and hydrogen peroxide (30\%) were purchased from Baker. Mercaptoundecanoic acid (95\%) (MUA), $\mathrm{N}$-hydroxysuccinimide (98\%) (NHS), $\mathrm{N}$-(3-dimethylaminopropyl)- $\mathrm{N}$ ethylcarbodiimide hydro-chloride (EDC), $\beta$-mercaptoethanol (MCE), sodium phosphate monobasic and sodium phosphate dibasic (PB), Ethanolamine (EA), $\mathrm{HCl}$ and Tween-20 were purchased from SigmaAldrich. Ultrapure water with a resistivity of $18.2 \mathrm{M} \Omega$ was used (Purelab). Tetramethyl benzidine (TMB) solution was purchased from Promega, and horseradish peroxidase (HRP) -conjugated rabbit anti-human IgG from Santa Cruz Biotechnology. a-Methoxy- $\omega$-mercapto PEG, (CH3-PEG-SH) MW: 750 Da (PEG) was purchased from Rapp Polymere (Germany).

\subsection{Peptides}

Unphosphorylated peptide: C- ${ }^{412}$ RIEEELGSKAKF ${ }^{423}$ (ENOAmer), concentration $600 \mu \mathrm{g} / \mathrm{mL}$; MW 1509,76-397.41 $\mu \mathrm{M}$. Phosphorylated peptide: $\mathrm{C}^{4}{ }^{413}$ RIEEELGS $\mathrm{KAKF}^{423}$ (phosphoENOAmer), concentration $1000 \mu \mathrm{g} / \mathrm{mL}$; MW 1587,68-629.84 $\mu \mathrm{M}$. The two solid phase chemically synthesized peptides (Primm srl, Milan, Italy) have the sequence of 13 aminoacid residues of the $\alpha$-enolase domain previously identified by LC-MS/MS analysis (Zhou et al., 2010) with the addition of a Cysteine to the Arginine (R) to allow peptide immobilization on the sensor surface. ENOAmer and phosphoENOAmer peptides differ only for the phosphorylation of Serine 419.

\subsection{Human serum}

The study was conducted with ethical approval from the Ethical Committees of the Department of Internal Medicine, University of Turin, San Giovanni Battista Hospital, Turin (authorization No. 0058870). Serum samples were isolated from venous blood at time of diagnosis with the informed consent of patients and healthy donors and stored at $-80^{\circ} \mathrm{C}$ until use. De-identified numeric specimen codes were used to protect the identity of the individuals. Diagnosis of PDAC was always confirmed by histological or cytological analysis. Three typologies of human serum samples were supplied: (1) serum pool derived from $(n=10)$ PDAC patients that does not contain antibodies to the two acidic phosphorylated isoforms of ENOA, here after referred to as ENOA1,2 $2^{-}$sera; (2) serum pool derived from $(n=10)$ PDAC patients, that contains antibodies to the two acidic phosphorylated isoforms of ENOA, here after referred to as ENOA $1,2^{+}$sera; (3) serum pool derived from $(n=10)$ healthy subjects without a prior history of cancer or autoimmune disease, here after referred to as HS sera. The reactivity of the sera against the two peptides was assessed by 2-DE WB (Tomaino et al., 2011) and ELISA (Novelli et al., 2011).

\subsection{Quartz crystal microbalance with dissipation monitoring (QCM-D) measurements}

QCM measurements were performed using the Q-Sense E1 system (Q-Sense, Sweden).

Briefly, the QCM-D technique (Rodahl et al., 1995; Vikinge et al., 2000; Hook et al., 2002) exploits a piezoelectric quartz crystal sandwiched between gold electrodes that starts to oscillate at its resonance frequency $(\sim 5 \mathrm{MHz})$ as a voltage is applied across the crystal. As molecules adsorb to the sensor surface, the resonance frequency changes and the adsorbed mass, $\Delta m$, can be calculated from the change in frequency, $\Delta f$, via the Sauerbrey equation (Sauerbrey, 1959):

$\Delta m=-\frac{C \times \Delta f}{n}$

Here, $n$ is the number of overtone $(n=1,3,5$ or 7 for $5 \mathrm{MHz}, \quad 15 \mathrm{MHz}, 25 \mathrm{MHz}$ and $35 \mathrm{MHz}$, respectively) and $C=17.7 \mathrm{ng} \mathrm{cm}^{-2} \mathrm{~Hz}^{-1}$ for the sensor crystal used in this study. This equation is applied with high accuracy to rigid films that exhibit no meaningful changes in dissipation signals.

The sensor crystals used were $5 \mathrm{MHz}, 14 \mathrm{~mm}$ diameter, AT-cut quartz discs with an evaporated gold surface (Q-Sense). The resonance frequency and energy dissipation were measured simultaneously at the fundamental frequency of the crystal (1st harmonic at $5 \mathrm{MHz}$ ) and six harmonics of the fundamental frequency (third, fifth, seventh, ninth, eleventh and thirteenth harmonic at $15 \mathrm{MHz}, 25 \mathrm{MHz}, 35 \mathrm{MHz}, 45 \mathrm{MHz}, 55 \mathrm{MHz}, 65 \mathrm{MHz}$, respectively). For simplicity, only changes in the mass increase, $\Delta m_{7}$, and dissipation, $\Delta D_{7}$, of the seventh overtone ( $35 \mathrm{MHz}$ ) were presented in the reported graphs. Measurements at natural frequency $(5 \mathrm{MHz})$ were not considered since the fundamental resonance is very sensitive to bulk solution changes and generates un-reliable data.

\subsubsection{Cleaning procedure}

Before each experiment, sensor chips were placed in a Diener Plasma cleaner (Pico model) for $10 \mathrm{~min}$ and immediately cleaned in the boiling mixture of $28-30 \%$ ammonium hydroxide, $30 \% \mathrm{H}_{2} \mathrm{O}_{2}$ and ultrapure water in a $1: 1: 5(v / v)$ ratio for $10 \mathrm{~min}$; then treated again with oxygen plasma for $1 \mathrm{~min}$, rinsed with ultrapure water and ethanol.

Preliminarily, we analyzed three different functionalization procedures of the quartz crystals, and hence, the most promising one was used to perform the planned experiments. Details on such methods are reported below.

\subsubsection{MCE ex-situ quartz crystal functionalization}

After cleaning, quartz crystal was immediately immersed into $0.7 \mathrm{mM}$ MCE solution for $2 \mathrm{~h}$. After 3-5-min washing cycles in ultrapure water to remove the un-adsorbed thiol compounds, quartz crystal was mounted inside QCM-D flow module.

\subsubsection{MUA/NHS/EDC ex-situ quartz crystal functionalization}

Alternatively, after cleaning, the quartz crystal was immediately immersed into a $10 \mathrm{mM}$ ethanol solution of MUA and kept overnight at room temperature $\left(20-25^{\circ} \mathrm{C}\right)$ in order to obtain 
the thiol self-assembled monolayer (SAM) on the gold surface. Afterwards, weakly interacting thiols were removed from the surface by rinsing with ethanol and ultrapure water. Then the crystal was incubated for $3 \mathrm{~h}$ into a freshly prepared aqueous mixture of $15 \mathrm{mM}$ NHS and $75 \mathrm{mM}$ EDC for activation of carboxyl groups.

\subsubsection{PEG ex-situ quartz crystal functionalization}

After cleaning, quartz crystal was immediately immersed into a $0.5 \mathrm{mM}$ DMF solution of PEG and kept overnight at room temperature $\left(20-25^{\circ} \mathrm{C}\right)$. After 2-5-min washing cycles in DMF and final washing in ultrapure water to remove the un-adsorbed thiol compounds, quartz crystal was mounted inside QCM-D flow module.

\subsubsection{Background signal estimation}

In order to estimate the background signal due to serum-SAM interaction, once the MCE, PEG or MUA/NHS/EDC functionalized crystals were inserted in the QCM-D chamber, avoiding surface dehydration, two different procedures were followed.

In the MCE and PEG cases, (1) the flow cell was filled with PB in continuous (at $100 \mu \mathrm{L} / \mathrm{min}$ ) to obtain stable $\Delta f$ and $\Delta D$ baseline signals, and after 30 min equilibration (2) $100 \mu \mathrm{L}$ of human serum pool were injected to completely replenish the flow cell. (3) Frequency $(\Delta f)$ and dissipation $(\Delta D)$ shifts were measured in real-time during pool injection and during the incubation time $(2 \mathrm{~h})$. At the end of incubation, (4) the final washing was performed at $400 \mu \mathrm{L} / \mathrm{min}$ with $0.5 \%$ Tween-20 for $15 \mathrm{~min}$, and by ultrapure water.

In the MUA/NHS/EDC case, two additional steps were introduced after step 1 . After $30 \mathrm{~min}$ equilibration, $1 \mathrm{M}$ EA solution was injected and incubated for $10 \mathrm{~min}$ in order to passivate the reactive surface. The reaction was followed by a rinsing with $\mathrm{PB}$ buffer, to remove not-reacted EA. The SAM formed on the QCM sensor surface named MUA/EA. Afterwards steps 2-4 were performed as described above. All experiments were carried out at $25{ }^{\circ} \mathrm{C}$ and replicated at least twice.

\subsubsection{Ex-situ quartz crystal functionalization with MUA/NHS/EDC/ peptide}

For peptide antigen immobilization, subsequently ultrapure water washing, the activated MUA/NHS/EDC sensor surface was incubated with $50 \mu \mathrm{L}$ of $200 \mathrm{nM}$ phosphoENOAmer or ENOAmer in $10 \mathrm{mM}$ phosphate buffer solution (PB, $\mathrm{pH} 7.4$ ), for $2 \mathrm{~h}$ at room temperature. The quartz crystal was once more washed with ultrapure water in order to remove weakly adsorbed molecules and immediately mounted in the QCM-D chamber ready to use, avoiding surface dehydration. Activated MUA is supposed to react with amino groups of the peptide and form amide bonds. Considering the specific amino acid sequence of the peptide (in $\mathrm{PB}, R$ and $K$ have charged amino groups, not so reactive towards carboxyl-activated groups) and the location of the free amino group at the $C$ residue, the formation of an amide bond between a MUA reactive SAM with the amino terminal group of the peptide, far from the phosphorylated serine, is highly probable. This should allow to immobilize the peptide on QCM crystal without inhibiting its interaction with the auto-antibody.

\subsubsection{Typical QCM-D immunosorbed assay}

A typical QCM immunosorbed assay experiment involved five main steps performed after ex-situ peptide adsorption (Fig. 1). Specifically, (A) PB flowing to stabilize the signals in liquid environment; (B) blocking of un-specific sites by injection and incubation with $1 \mathrm{M} \mathrm{EA}$; (C) rinsing with $\mathrm{PB}$ to remove not-reacted $\mathrm{EA}$; (D) injection and incubation of serum for $2 \mathrm{~h} 50 \mathrm{~min}$; (E) washing procedure with $0.5 \%$ Tween-20 and $(\mathrm{F})$ ultrapure water.

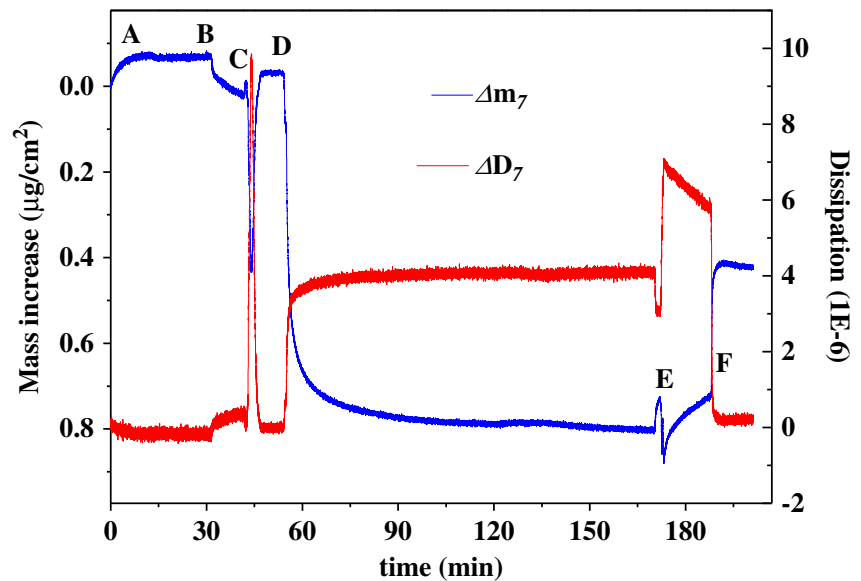

Fig. 1. Example of raw data from a QCM-D experiment. The time-dependent mass adsorption (blue curve) and dissipation shifts (red curve) recorded during the immobilization of human serum on the functionalized surface of quartz crystal: (A) PB flowing; (B) blocking of un-specific sites by $1 \mathrm{M} \mathrm{EA}$; (C) rinsing with $10 \mathrm{mM}$ PB to remove not-reacted EA; (D) injection and incubation of serum; (E) washing procedure with $0.5 \%$ Tween-20 and (F) ultrapure water. (For interpretation of the references to color in this figure legend, the reader is referred to the web version of this article.)

To evaluate the efficiency of the designed phosphoENOAmer probe immobilization, in terms of interaction with the ENOA specific serum immunoglobulins, we performed experiments using ENOA1,2 $2^{+}$sera; consistent results were compared with those obtained from negative control assays carried on testing ENOA1,2- as well as on HS sera. Similarly, as an internal control, ENOAmer - serum interaction was evaluated expecting none, or low, IgG specific binding.

The original data were analyzed by Origin 8 software (OriginLab Corporation, Northampton, MA), Q-Soft and Q-Tools (Q-Sense). QCM experiments were repeated at least twice, for each measurement. In the QCM sensorgrams, we reported the behavior of mass increase and dissipation of the overtone 7 as example. The shifts were reported in the tables as mass changes $(\Delta \mathrm{m})$, calculated from the sensorgrams, applying the Sauerbrey equation (par. 2.4). Although that equation is not strictly true for adsorption of proteins, it is used as an approximation to compare relative amounts of proteins adsorbed on the crystals coated with different SAMs (Wong et al., 2012). Data are reported as an average over five overtones; the reported errors are calculated as standard deviation.

\subsection{AFM analysis}

Atomic Force Microscopy (AFM) in tapping mode (Nanoscope $\mathrm{V}$, Bruker Inc.) was used to characterize the morphology of the MCE, PEG and MUA/EA SAM-modified quartz crystal surfaces at a micro-scale level. For these experiments, we used silicon cantilevers (model RFESPA) with a tip radius of $8 \mathrm{~nm}$ and a resonance frequency of $70-85 \mathrm{kHz}$. Images at $3 \times 3 \mu \mathrm{m}$ were acquired at $256 \times 256$ resolution.

\subsection{Contact angle measurements}

Dynamic advancing $\left(\theta_{a}\right)$ and receding $\left(\theta_{r}\right)$ water contact angles on quartz crystals were measured using a CAM 200 (KSV Instruments Ltd., Finland) instrument. $\theta_{a}$ and $\theta_{r}$ were obtained by adding or withdrawing $3 \mu \mathrm{l}$ of water with a rate of $3 \mu \mathrm{l} / \mathrm{s}$ for 4 cycles separated by $1 \mathrm{~s}$ at room temperature $\left(22^{\circ}\right.$ to $\left.23^{\circ} \mathrm{C}\right)$. The values reported are an average of four measurements and the errors are calculated as standard deviations from the medium value. 


\section{Results and discussion}

\subsection{Background signal estimation}

QCM is a mass-based biosensor and its resolution is strongly affected by the adsorption of biomolecules different from the specific immunoglobulins (directed against the phosphoENOAmer peptide) that are components of the complex serum matrixes. In order to minimize the un-specific interactions, it is important to properly functionalize the crystal surface with ordered selfassembled monolayers (SAMs) and to select high yield binding conditions (in terms of detergents, ionic strength and serum dilution). Initially we have performed some tests to define a SAM structure in order to inhibit ENOA1,2 ${ }^{+}$sera un-specific adsorptions.

SAMs able to link with high specificity an antibody dispersed in a "protein-repellent" SAM, originate surfaces that favor the specific biomolecular interactions of interest avoiding non-specific binding events (Ostuni et al., 1999; Gronbeck et al., 2000; Ostuni et al., 2001). Among a large number of possible SAMs, MCE is often used because the mercapto moiety allows to anchor the molecule directly to the gold surface and the polar $-\mathrm{OH}$ head reduces unfavorable biomolecular interactions (Tichoniuk et al., 2010).

Therefore, we have functionalized the electrode surface as described in Section 2.4.2 and performed experiments as described in Section 2.4.5. Different dilutions of ENOA1,2 ${ }^{+}$sera interacting with the sensor surface, were evaluated and the results summarized in Table 1.

The first experiment was performed using ENOA1,2 ${ }^{+}$sera diluted 1:50 in PB. In the sensorgram (data shown in Fig. S1) the serum pool adsorption to the MCE quartz crystal surface is observed as a quick frequency drop (indicating a mass increase) followed by a slower decrease as the adsorption saturates. The mass increase $\left(\Delta m_{1}\right)$, calculated by estimating the frequency signal $1 \mathrm{~min}$ prior to the serum pool injection and after pool injection signal reached the saturation, was calculated to be $1.292 \pm 0.018 \mu \mathrm{g} / \mathrm{cm}^{2}$. The mass increase after washing $\left(\Delta m_{2}\right)$, calculated from the difference between the frequency signal $1 \mathrm{~min}$ prior to the serum pool injection and at the end of the ultrapure water washing, was evaluated to be $1.064 \pm 0.012 \mu \mathrm{g} /$ $\mathrm{cm}^{2}$. The high background signal before and after washing indicated a great nonspecific adsorption of serum components.

A second experiment was performed using ENOA1,2 ${ }^{+}$sera diluted $1: 50$ in $\mathrm{PB}$, additioned of $0.5 \%$ of Tween-20, since detergents seem to reduce nonspecific adsorption of serum proteins (Smith et al., 1978). In this case, a $\Delta m_{1}$ and $\Delta m_{2}$ of $1.274 \pm 0.036 \mu \mathrm{g} / \mathrm{cm}^{2}$ and $0.789 \pm 0.014 \mu \mathrm{g} / \mathrm{cm}^{2}$ were calculated, due to the decrease of non-specific adsorption of serum components in presence of detergents.

A better result was obtained by further dilution of the serum pool: the $\Delta m_{1}$ and $\Delta m_{2}$ for a 1:100 dilution decreased of $0.336 \mu \mathrm{g} / \mathrm{cm}^{2}$ and $0.168 \mu \mathrm{g} / \mathrm{cm}^{2}$, respectively, compared with a 1:50 dilution.

However, despite all the attempts to decrease the un-specific adsorptions, we noted that the interaction of the SAM with

Table 1

Background signal determination in QCM-D measurements for ENOA1,2 ${ }^{+}$sera. $\Delta m_{1}$ represents the mass adsorbed by the crystal soon after pool injection; $\Delta m_{2}$ is the mass increase after the last washing with water.

\begin{tabular}{llll}
\hline SAM & Pool dilution and additives & $\Delta \boldsymbol{m}_{\mathbf{1}}\left(\boldsymbol{\mu g} / \mathbf{c m}^{\mathbf{2}}\right)$ & $\boldsymbol{\Delta} \boldsymbol{m}_{\mathbf{2}}\left(\boldsymbol{\mu g} / \mathbf{c m}^{\mathbf{2}}\right)$ \\
\hline MCE & $1: 50$ & $1.292 \pm 0.018$ & $1.064 \pm 0.012$ \\
MCE & $1: 50 /$ Tween-20 & $1.274 \pm 0.036$ & $0.789 \pm 0.014$ \\
MCE & $1: 100 /$ Tween-20 & $0.938 \pm 0.018$ & $0.621 \pm 0.014$ \\
MUA/EA & $1: 100 /$ Tween-20 & $0.442 \pm 0.036$ & $0.221 \pm 0.003$ \\
PEG & $1: 100 /$ Tween-20 & $0.796 \pm 0.194$ & - \\
\hline
\end{tabular}

ENOA1,2 $2^{+}$sera was still too strong to achieve a reasonable QCM sensitivity. Therefore we have developed a different functionalization strategy based on a MUA SAM (Ayela et al., 2007; Hong et al., 2009; Deng et al., 2006; Hao et al., 2009). As described in the previous paragraph, we have used NHS/EDC to activate SAM carboxyl groups and added EA to block reactive sites on the gold surface. In this experiment, the $\Delta m_{1}$ and $\Delta m_{2}$ significantly decreased (of $0.496 \mu \mathrm{g} / \mathrm{cm}^{2}$ and $0.4 \mu \mathrm{g} / \mathrm{cm}^{2}$, respectively if compared with MCE 1:100 dilution) for the ENOA1,2 ${ }^{+}$sera test, revealing a strong decrease of non-specific serum components adsorption. Similar background signals were found for ENOA1,2 and HS sera. These results demonstrate that an EA passivation is more resistant to serum protein adsorption than a MCE one and that serum dilution as well as addition of Tween-20 decrease unspecific interactions.

With the aim of further decreasing the background signal, we functionalized the QCM surface with a thiolated-PEG, that is well known for its capability to decrease the non-specific adsorption of proteins (Love et al., 2005). PEG SAMs can be adequately functionalized by photochemistry (Kim et al., 2009); alternatively, carboxylated PEGs to be activated with NHS/EDC are available (Ayela et al., 2007). From the data reported in Table 1, it seems that no improvements are associated to the use of PEG SAMs for our experiments since a background signal $\Delta m_{1}$ of $0.796 \pm$ $0.194 \mu \mathrm{g} / \mathrm{cm}^{2}$ was calculated. Additionally, due to strong instabilities during washing, it was not possible to quantify the mass increase $\Delta m_{2}$.

In order to explain the origin of the different interactions of these three SAMs with sera, we performed studies of dynamic contact angle.

Dynamic contact angle measurements were carried out to determine the advancing and receding contact angles of the SAMs. The contact angle hysteresis $\theta_{\mathrm{i}}$, the difference between advancing $\theta_{\mathrm{a}}$ and receding $\theta_{\mathrm{r}}$ contact angles, measures the adhesion of the liquid droplet to the surface and is influenced by surface heterogeneities (Johnson and Dettre, 1964; Wang et al., 2011). A small contact angle hysteresis is consistent with a surface that is homogeneous and smooth, and a large hysteresis implies a heterogeneous surface.

We have measured $\theta_{\mathrm{i}}$ for a bare quartz crystal and found an angle of $\sim 42^{\circ}$, in agreement with previous measurements (Stadler et al., 2003). The functionalization with SAMs decreased the hysteresis angle as shown in Table 2. MUA/EA SAMs exhibited a high hysteresis; a lower value was calculated for MCE. The lowest hysteresis was found for PEG SAMs. From a comparison with QCM measurements (Table 1), we observed that the capability to decrease adsorption from sera was directly proportional to the hysteresis. High hysteresis SAMs were, indeed, associated to lower protein adsorption. This conclusion is not un-expected since there are several works in which proteins and blood plasma adhesion was reduced on high hysteresis surfaces (Wong et al., 2012 and ref. ivi cited)

This result was attributed to presence of nanoscale segregation of hydrophobic/hydrophilic domains. To explain the anti-fouling properties of heterogeneous surfaces it was proposed that a set of residues on the protein molecule forms the initial contact with

Table 2

Dynamic contact angle measurements of quartz crystals functionalized with SAMs of MCE, MUA and PEG compared with the bare substrate.

\begin{tabular}{lccr}
\hline QCM & $\boldsymbol{\theta}_{\mathbf{a}}\left({ }^{\circ}\right)$ & $\boldsymbol{\theta}_{\mathbf{r}}\left({ }^{\circ}\right)$ & $\boldsymbol{\theta}_{\mathbf{i}}\left({ }^{\circ}\right)$ \\
\hline Bare Au & $71 \pm 1$ & $29 \pm 1$ & $42 \pm 2$ \\
MUA/EA SAM & $61 \pm 1$ & $29 \pm 2$ & $32 \pm 3$ \\
MCE SAM & $58 \pm 2$ & $38 \pm 2$ & $20 \pm 4$ \\
PEG SAM & $58 \pm 1$ & $51 \pm 2$ & $7 \pm 3$ \\
\hline
\end{tabular}


a

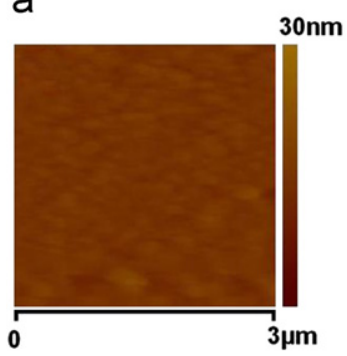

e

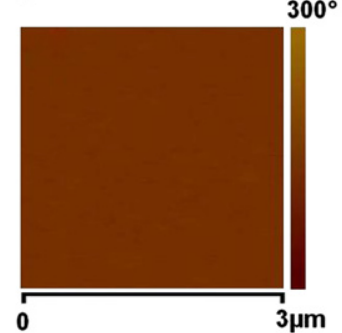

b

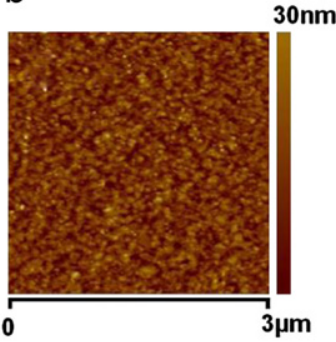

$f$

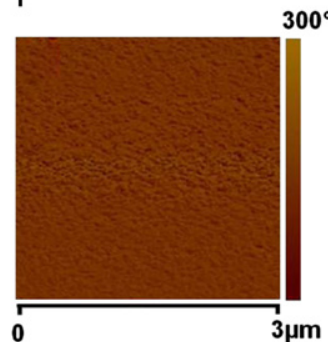

C

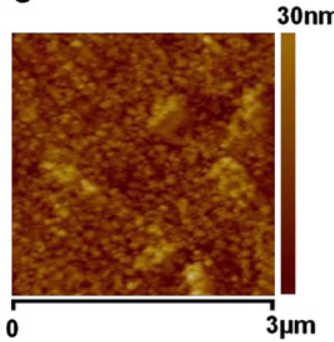

9

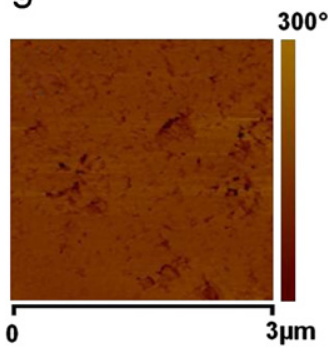

d

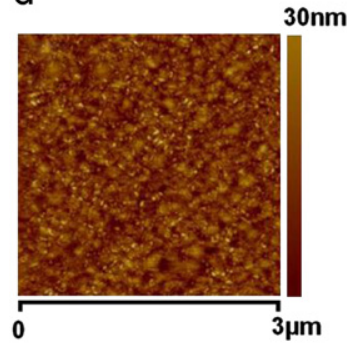

h

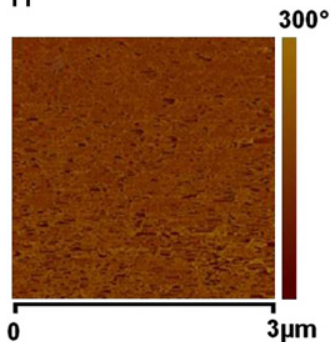

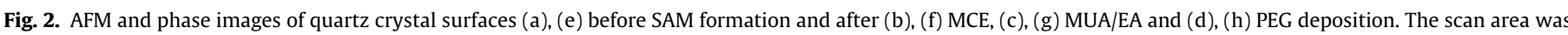
$3 \times 3 \mu \mathrm{m}^{2}$.

the surface, followed by additional contacts due to cooperative effects from neighboring residues (Macritchie, 1978). A surface with molecular-scale heterogeneities inhibits protein adsorption since it disrupts the initial adsorption event.

In the perspective of demonstrating the presence of such domains on the surface of quartz crystals covered with MUA/EA SAMs, we performed AFM analysis with phase contrast. From the images shown in Fig. 2, phase shift contrasts, suggesting nanoscale segregation can be observed. The contrasts appeared quite clear for MUA/EA SAMs and more noisy for the other two SAMs. No phase contrast was observed on the bare crystal surface. The presence of an evident alternation of domains in MUA/EA SAMs maybe attributed to nanoscale segregation of hydrophobic/hydrophilic areas, probably associated to the long $\mathrm{C}$ aliphatic chain and the $-\mathrm{OH}$ or $-\mathrm{COOH}$ groups of MUA and EA molecules.

After the background signal optimization, we performed QCM measurements of autoantibody response to ENOA-derived peptides using a QCM crystal functionalized as described in section 2.4.7.

\subsection{Analysis of frequency changes at different peptide-serum pool interactions}

QCM experiments performed using the two phosphoENOAmer and ENOAmer peptides interacting with ENOA1,2 $2^{+}, \mathrm{ENOA} 1,2^{-}$and HS sera are shown in Fig. 3; for a better comparison, only serum adsorption and washing steps (corresponding to steps D-F of Fig. 1) are visualized. Before discussing these results, it is important to remember that ENOA1,2 $2^{-}$sera and HS sera don't contain antibodies against the phosphorylated $\alpha$-enolase, but there are probably other immunoglobulins with a plausible affinity for both the peptides. Only ENOA1,2 $2^{+}$sera have antibodies against the phosphorylated form of $\alpha$-enolase that would allow to form specific bonds with the synthesized phosphoENOAmer (Tomaino et al., 2007).

Fig. $3 a$ and $b$ shows the interaction of the two peptides with HS and ENOA $1,2^{+}$sera, respectively. Fig. $3 c$ represents peptides interaction with ENOA $1,2^{-}$sera. After sera injections, an initial rapid frequency decrease (mass adsorption on the crystal surface) followed by a steady-state due to saturation was observed. Upon exchange of the sera solution to a pure buffer solution a frequency increase (desorption at the liquid/crystal interface) occurred.

Assuming that the EA monolayer doesn't adsorb specifically any molecule of the human serum, we found a difference in the interactions between both un-phosphorylated and phosphorylated peptides with each serum pool. HS and ENOA $1,2^{+}$sera seem to interact with phosphoENOAmer peptide more than with ENOAmer one; on the contrary, ENOA $1,2^{-}$sera interact strongly with ENOAmer peptide and less with phosphoENOAmer, confirming WB (Tomaino et al., 2011) and ELISA (Novelli et al., 2011) results. This different behavior was quantified by the mass changes $\Delta \Delta m$ (difference between $\Delta m$ (phosphoENOAmer) $-\Delta m$ (ENOAmer)).

The $\Delta \Delta m$ values for each serum are reported in Table 3 . As it can be observed, $\Delta \Delta m$ for HS sera is about $0.12 \pm 0.07 \mu \mathrm{g} / \mathrm{cm}^{2}$; this value is doubled in the case of ENOA $1,2^{+}$sera and it is negative for ENOA $1,2^{-}$sera. The difference between HS and ENOA $1,2^{+}$sera is meaningful and repeatable-a second set of measurements of ENOA $1,2^{+}$sera resulted in a $\Delta \Delta m$ of $0.230 \pm 0.012 \mu \mathrm{g} / \mathrm{cm}^{2}$.

A preferable interaction of HS sera with PhosphoENOAmer seems to occur but we know that no autoantibodies to phosphorylated ENOA1,2 $2^{+}$are present in HS sera. Therefore, in order to understand the difference between the layers formed at the peptide-sera interface we studied the dissipation signal in comparison with the frequency shift.

Usually, the observed changes on the sensor of the QCM-D can give information on the viscoelasticity properties of the layer. Using the simultaneously measured $\Delta f_{7}$ versus time and the $\Delta D_{7}$ versus time responses, we plotted $\Delta D_{7}$ versus $\Delta f_{7}$ to study the interaction of $\mathrm{HS}, \mathrm{ENOA} 1,2^{+}$and ENOA1,2- sera with the two peptide layers.

Fig. 4 presents these results for different sera-phosphoENOAmer interactions. Similar curves (data shown in Fig. S2) were acquired for other sera-peptides combinations. A slope was calculated for each curve, indicative of the influence of serum on viscoelasticity properties of the peptide/MUA/EA SAM. The slope values for the curves are reported in Table 4. Indeed, a small value of the slope $K$ $(K=\Delta D / \Delta f)$ reveals a strong and compact film (Ayela et al., 2007). It is noteworthy that the smallest $K$ value $(0.0463 \pm 0.0001)$ was obtained in the case of phosphoENOAmer-ENOA1,2 $2^{+}$film. Higher $K$ values (between 0.08 and 0.19 ) were calculated for all the other 
a

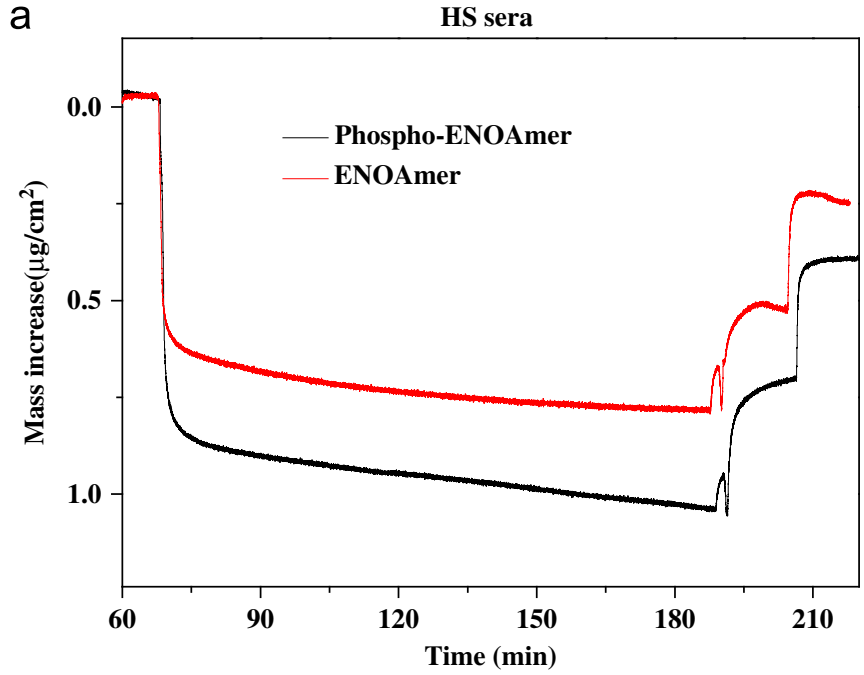

b

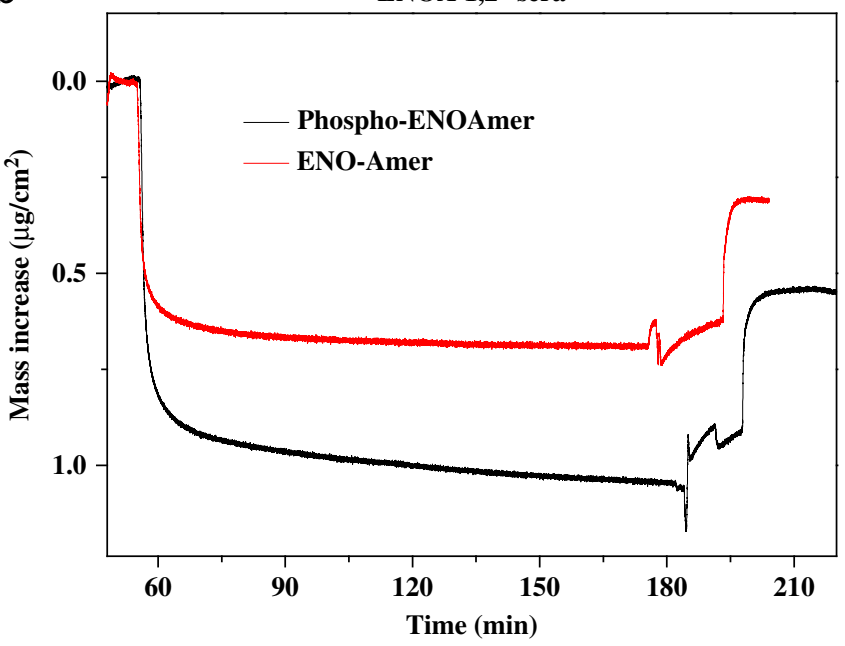

C

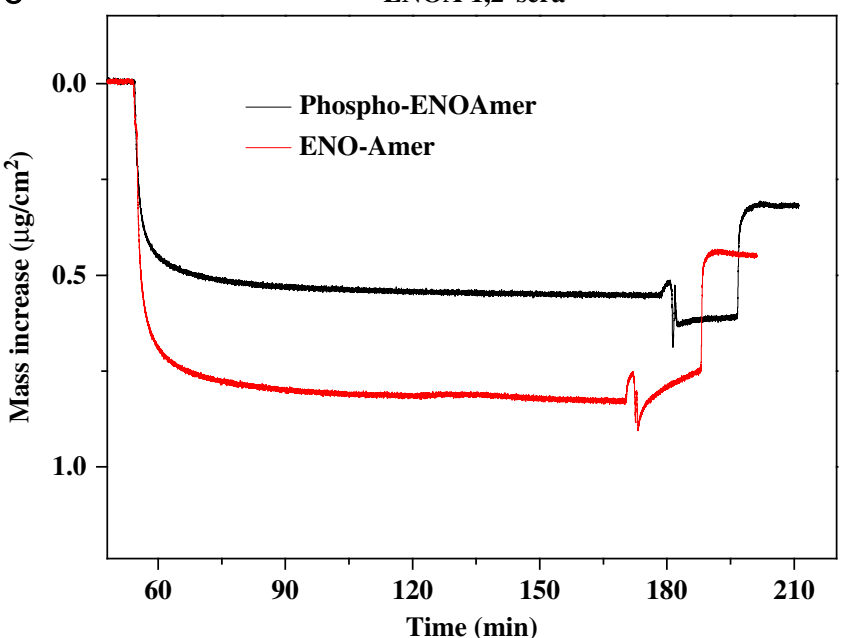

Fig. 3. QCM analysis. Typical time-course plots of the mass changes due to phosphoENOAmer (black curve) and ENOAmer (red curve) with (a) HS, (b) ENOA1,2+ and (c) ENOA1,2- sera.

cases, meaning that more disordered and less compact films were formed at the crystal interface. We believe that specific interactions produce more ordered antibodies films than un-specific ones. Therefore, we derive that phosphoENOAmer forms specific
Table 3

Quantification of the peptide-serum pool interactions by QCM. $\Delta \Delta \mathrm{m}$ is defined as the difference between $\Delta \mathrm{m}$ (PhosphoENOAmer) and $\Delta \mathrm{m}$ (ENOAmer)

\begin{tabular}{lllr}
\hline Sera & $\begin{array}{l}\Delta \boldsymbol{m} \\
(\text { PhosphoENOAmer }) \\
\left(\boldsymbol{\mu g} / \mathbf{c m}^{2}\right)\end{array}$ & $\begin{array}{l}\Delta \boldsymbol{\Delta m}(\mathbf{E N O A m e r}) \\
\left(\boldsymbol{\mu g} / \mathbf{c m}^{2}\right)\end{array}$ & $\Delta \boldsymbol{\Delta m}\left(\boldsymbol{\mu g} / \mathbf{c m}^{2}\right)$ \\
\hline HS sera & $0.409 \pm 0.035$ & $0.290 \pm 0.035$ & $0.12 \pm 0.07$ \\
ENOA1,2+sera & $0.557 \pm 0.012$ & $0.310 \pm 0.007$ & $0.247 \pm 0.019$ \\
ENOA1,2-sera & $0.198 \pm 0.009$ & $0.255 \pm 0.007$ & $-0.057 \pm 0.016$ \\
\hline
\end{tabular}

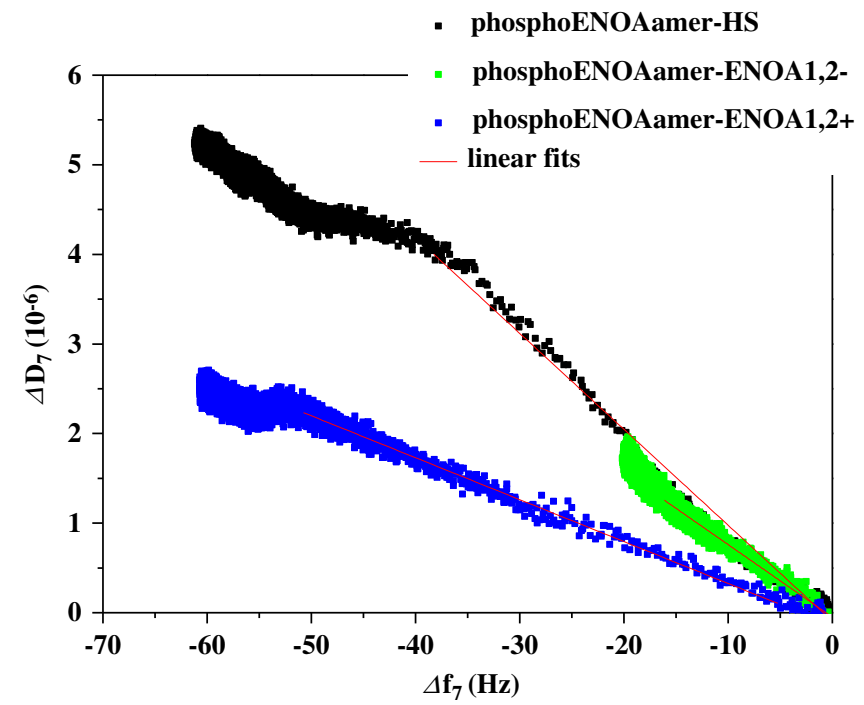

Fig. 4. $\Delta \mathrm{D} 7$ versus $\Delta \mathrm{f} 7$ plots for phosphoENOAmer-HS sera (black dots), phosphoENOAmer-ENOA1,2 - sera (green dots), phosphoENOAmer-ENOA1,2 + sera (blue dots) and related linear fits (red lines). (For interpretation of the references to color in this figure legend, the reader is referred to the web version of this article.)

Table 4

$K$ values calculated as slope of a linear fit of the graphs shown in Fig. 4 and in Fig. S2.

\begin{tabular}{lc}
\hline Peptide-sera interactions & $\boldsymbol{K}$ \\
\hline $\begin{array}{l}\text { PhosphoENOAmer-HS } \\
\text { PhosphoENOAmer- }\end{array}$ & $0.1070 \pm 0.0003$ \\
$\quad$ ENOA1,2- & $0.0809 \pm 0.0005$ \\
PhosphoENOAmer- & $\mathbf{0 . 0 4 6 3} \pm \mathbf{0 . 0 0 0 1}$ \\
$\quad$ ENOA1,2 & \\
ENOAmer-HS & $0.191 \pm 0.002$ \\
ENOAmer-ENOA1,2 & $0.091 \pm 0.001$ \\
ENOAmer-ENOA1,2 & $0.125 \pm 0.002$ \\
MUA-ENOA1,2 & $0.1000 \pm 0.0002$ \\
\hline
\end{tabular}

interactions with ENOA1,2+ antibodies; on the contrary, phosphoENOAmer interacts non-specifically with immunoglobulin present in HS and ENOA1,2 $2^{-}$sera. Similar $K$ values were calculated for passivated MUA surfaces (EA SAMs) and ENOA1,2+ sera in absence of peptides. ENOAmer interacts by forming very disordered layers (with $K \geq 0.11$ ) with HS and ENOA1,2 ${ }^{+}$sera. Weaker interactions probably drive ENOAmer-sera antibodies assemblies.

In conclusion, QCM-D measurements allow us to confirm that phosphoENOAmer is an appropriate probe able to discriminate between specific interactions with ENOA1,2+ antibodies and nonspecific interactions with other immunoglobulins present in the sera of ENOA1,2- patients or HS. Additionally, these experiments demonstrate that QCM-D could be a sensitive biosensor for studying antigen-antibody interactions as well as a tool to establish the less reactive surface to non-specific binding with the final target to 
develop dedicated microplates functionalization protocols for detecting PDAC markers with un-labeled biosensors.

\section{Conclusion}

A study of PDAC serum reactivity against phosphorylated peptides using QCM technique was carried out. The frequency shift as well as dissipation signals were used as analytical signal for qualitative analysis of the serum pool derived from PDAC adsorption to the peptide-functionalized surfaces. The peptide-based quartz crystal microbalance biosensor allows a simple flow injection procedure for rapid screening and selective assay of human serum pools.

The results obtained indicate that the peptide probes here used have sufficient discriminatory power to detect specific serum pool components. Therefore the use of QCM-D is very promising tool to monitor and study human serum interactions with peptides as well as to produce surfaces less sensitive to unspecific interactions in the perspective of suggesting specific protocols for detecting PDAC markers with label-free biosensors.

\section{Acknowledgments}

The authors acknowledge the EU project "ROC", grant agreement n. 213803 for financial support. This work was supported in part from regione Puglia: Progetto Strategico (PS105) and Progetto Reti di Laboratori (NaBiDit) and from the European Community, Seventh Framework Program European Pancreatic Cancer-TumorMicroenvironment Network (EPC-TM-Net, nr. 256974); Associazione Italiana Ricerca sul Cancro (AIRC) $5 \times 1000$ (no. 12182) and IG (nrs. 5548 and 11643); Ministero della Salute: Progetto Integrato Oncologia; Regione Piemonte: Ricerca Industriale e Sviluppo Precompetitivo (BIOPRO and ONCOPROT), Ricerca Industriale "Converging Technologies" (BIOTHER), Progetti strategici su tematiche di interesse regionale o sovra regionale (IMMONC), Ricerca Sanitaria Finalizzata, Ricerca Sanitaria Applicata; Ministero dell'Istruzione e della Ricerca (MIUR), Progetti di Rilevante Interesse Nazionale (PRIN 2009); University of Turin-Progetti di Ateneo 2011: Mechanisms of REsistance to anti-angiogenesis regimens THErapy (grant Rethe-ORTO11RKTW). MC is recipient of a fellowship from Fondazione Italiana Ricerca sul Cancro (FIRC).

\section{Appendix A. Supporting information}

Supplementary data associated with this article can be found in the online version at http://dx.doi.org/10.1016/j.bios.2012.10.012.

\section{References}

Ayela, C., Roquet, F., Valera, L., Granier, C., Nicu, L., Pugnière, M., 2007. Biosensors and Bioelectronics 22 (12), 3113-3119.

Butler, J.E., 2000. Methods 22, 4-23.
Chou, S.F., Hsu, W.L., Hwuang, J.M., Chen, C.Y., 2002. Clinical Chemistry 48 913-918.

Curie, J., Curie, P., 1880. Rendu. 91, 294-297.

Capello, M., Ferri-Borgogno, S., Cappello, P., Novelli, F., 2011. FEBS Journal 278 (7) 1064-1074.

Cappello, P., Tomaino, B., Chiarle, R., Ceruti, P., Novarino, A., Castagnoli, C., Migliorini, P., Perconti, G., Giallongo, A., Milella, M., Monsurrò, V., Barbi, S. Scarpa, A., Nisticò, P., Giovarelli, M., Novelli, F., 2009. The International Journal of Cancer 125, 639-648.

Deng, T., Li, J.S., Huan, S.Y., Yang, H.F., Wang, H., Shen, G.L., Yu, R.Q., 2006 Biosensors and Bioelectronics 21 (8), 1545-1552.

Gregorius, K., Mouritsen, S., Henrik, I., 1995. Journal of Immunological Methods $181(1), 65-73$

Gronbeck, H., Curioni, A., Andreoni, W., 2000. Journal of the American Society 122 3839-3842.

Hao, R., Wang, D., Zhang, X., Zuo, G., Wei, H., Yang, R., Zhang, Z., Cheng, Z., Guo, Y., Cui, Z., Zhou, Y., 2009. Biosensors and Bioelectronics 24 (5), 1330-1335.

Hidalgo, M., 2010. The New England Journal of Medicine 362, 1605-1617.

Hook, F., Voros, J., Rodhal, M., Kurrat, R., Boni, P., Ramsden, J.J., Textor, M., Spencer N.D., Tengvall, P., Gold, J., Kasemo, B., 2002. Colloids and Surfaces B: Biointerfaces 24 (2), 155-170.

Hong, S.R., Choi, S.J., Jeong, H.D., Hong, S., 2009. Biosensors and Bioelectronics 24 (6), 1635-1640.

Ivanov, V.S., Suvorova, Z.K., Tchikin, L.D., Kozhich, A.T., Ivanov, V.T., 1992 J. Immunological Methods 153 (1-2), 229-233.

Johnson, R.E.J., Dettre, R.H., 1964. Journal of Physical Chemistry 68, 1744.

Kim, D.-N., Lee, W., Koh, W.-G., 2009. Journal of Chemical Technology and Biotechnology 84, 279-284.

Love, J.C., Estroff, L.A., Kriebel, J.K., Nuzzo, R.G., Whitesides, G.M., 2005. Chemistry Reviews 105, 1103-1169.

Macritchie, F., 1978. Advances in Protein Chemistry 32, 283-326.

Novelli, F., Tomaino, B., Cappello, P., 2011. WIPO Patent Application. WO/2011/ 030302.

Ostuni, E., Yan, L., Whitesides, G.M., 1999. Colloids and Surfaces B: Biointerfaces $15,3-30$.

Ostuni, E., Chapman, R.G., Holmlin, R.E., Takayama, S., Whitesides, G.M., 2001. Langmuir 17, 5605-5620.

Rodahl, M., Hook, F., Krozer, A., Brzezinski, P., Kasemo, B., 1995. Review of Scientific Instruments 66 (7), 3924-3930.

Sauerbrey, G., 1959. Zeitschrift Fuer. Physik 155 (2), 206-222.

Shons, A., Dorman, F., Najarian, J., 1972. Journal of Biomedial Materials Research 6 (6), 565-570

Smith, J.A., Hurrell, J.G.R., Leach, S.J., 1978. Analytical Biochemistry 87, 299-305.

Stadler, H., Mondon, M., Ziegler, C., 2003. Analytical and Bioanalytical Chemistry $375,53-61$.

Su, X.L., Li, Y., 2004. Biosensors and Bioelectronics 19, 563-574.

Tichoniuk, M., Gwiazdowska, D., Ligaj, M., Filipiak, M., 2010. Biosensors and Bioelectronics 26, 1618-1623.

Tomaino, B., Cappello, P., Capello, M., Fredolini, C., Ponzetto, A., Novarino, A. Ciuffreda, L., Bertetto, O., De Angelis, C., Gaia, E., Salacone, P., Milella, M. Nisticò, P., Alessio, M., Chiarle, R., Giuffrida, M.G., Giovarelli, M., Novelli, F. 2007. Journal of Proteome Research 6, 4025-4031.

Tomaino, B., Cappello, P., Capello, M., Fredolini, C., Sperduti, I., Migliorini, P., Salacone, P. Novarino, A., Giacobino, A., Ciuffreda, L., Alessio, M., Nistico‘, P. Scarpa, A., Pederzoli, P., Zhou, W., Petricoin, E.F., Liotta, L.A., Giovarelli, M., Milella, M., Novelli, F., 2011. Journal of Proteome Research 10, 105-112.

Vikinge, T.P., Hansson, K.M., Sandstrom, P., Liedberg, B., Lindahl, T.L., Lundstrom, I. Tengvall, P., Hook, F., 2000. Biosensors and Bioelectronics 15, 605-613.

Wang, H., Ren, J., Hlaing, A., Yan, M., 2011. Journal of Colloid and Interface Science 354, 160-167.

Wong, S.Y., Han, L., Timachova, K., Veselinovic, J., Hyder, M.N., Ortiz, C., Klibanov, A.M., Hammond, P.T., 2012. Biomacromolecules 13, 719-726.

Wu, Z.Y., Shen, G.L., Wang, S.P., Yu, R.Q., 2003. Analytical Sciences 19, 437-440.

Zhou, W., Capello, M., Fredolini, C., Piemonti, L., Liotta, L.A., Novelli, F., Petricoin, E.F., 2010. Journal of Proteome Research 9, 2929-2936. 